\title{
Current Developments in Robotics and Mobile Machines
}

\author{
Massimo Callegari · Primo Zingaretti
}

Published online: 14 October 2015

(C) Springer Science+Business Media Dordrecht 2015

Mechatronics is a key interdisciplinary field where the most important breakthrough technologies are developed and then integrated in a wide variety of products, ranging from research laboratories down to consumer goods and real life applications. Robotics is one of the most visible instances of mechatronics and it is therefore liable of continuous improvements and changes of research perspectives; therefore it may be useful once and then to try summarizing some important results that are attained by the community of robotics researchers in their specific fields.

The goal of this special issue is to contribute presenting the latest results of researches in advanced robotics and mobile machines by a selected group of top researchers and scientists of different geographic regions and scientific interests. To this aim we have asked some researchers that presented their work at the $10^{\text {th }}$ IEEE/ASME Mechatronics and Embedded Systems and Applications Conference held in Senigallia (Italy) at the end of 2014 to prepare

M. Callegari $(\square)$

Department of Industrial Engineering \& Mathematical

Sciences, Università Politecnica delle Marche,

Ancona, Italy

e-mail: m.callegari@univpm.it

P. Zingaretti

Department of Information Engineering,

Università Politecnica delle Marche,

Ancona, Italy

e-mail: p.zingaretti@univpm.it an extended version of their papers that contained the new results obtained in the meantime. Thirteen papers have been selected out of the twenty-eight that had been presented at the specific Symposium held within the Conference. The received contributions have been reviewed by at least three international reviewers with expertise in the specific field and after a two stage reviewing process, eight articles have been finally retained and collected in the present special issue.

The main topics addressed by these papers are: design of robots and actuators, mobile robots, underwater robotics, industrial robotics, robotic vision.

Fei Chen et al. studied some error recovery methods to be used in the automatic mating of electronic connectors when a jamming occurs: four different strategies have been devised and experimentally tested and the related results are explained.

Giovanni Boschetti revises the fixtures currently available to sort items in automated assembly that encompass the limits of vibratory bowl feeders; then he shows how to take the maximum advantage of the most innovative rotating feeders, which requires a proper path planning technique to be implemented on the manipulator. The proposed strategy is tested with good results on an industrial off-the-shelf manipulator.

Luca Bruzzone and Pietro Fanghella present the second version of the well-known Mantis hybrid legwheel mobile robot, conceived for surveillance and inspection tasks in unstructured indoor and outdoor 
environments. A re-design of the legs with the introduction of auxiliary passive wheels is able to prevent the slip in the last phase of step climbing previously shown under some conditions.

Alessandro Cammarata and Rosario Sinatra explain the steady-state model of a towfish designed to monitor the marine pollution in the Mediterranean Sea between Malta and Sicily. A GUI interface has been developed to analyze, by means of computer simulations, the equilibrium conditions when some relevant parameters are varied and for the attitude control in underwater maneuvers.

Gundula Runge et al. propose a ball wheel mechanism with interesting features like slip measurement, friction sensing and free-wheel mode. Based on such drive, the design of an omnidirectional mobile robot platform driven by three redundant ball wheel units is shown in the article and the related kinematic model is provided. The motion planning for an individual ball drive is demonstrated by a real time algorithm and the good motion characteristics of the ball drive mechanism are demonstrated by means of experimental tests.

Matteo-Claudio Palpacelli et al. designed and patented a lockable spherical joint, which is intended to be manually or automatically configured in different kinematic solutions. In fact the device is conceived for being used as a conventional spherical joint or converted in a universal joint, or still downgraded to a revolute pair. A relevant use of the concept is shown with reference to a reconfigurable parallel kinematic machine, able to switch its kinematics capabilities from translating to spherical motions.

Massimo Callegari et al. designed a prototypal parallel kinematics machine that can be used for the orientation of parts or devices: the peculiarity of this manipulator is that, by exploiting non-holonomic constraints, it is able to bring the end-effector in any 3 d.o.f.'s orientation within its working space by only using 2 actuators.

Gianluca Di Fulvio et al. designed a multi-point stereo measurement system for quality control in industrial robotics applications by means of a single camera mounted on a six-axis manipulator. The solution proposed is low cost, flexible and guarantees a continuous movement without the need for stop\&go phases. Tested in a real industrial application, provides better results, in terms of accuracy and measurement speed, when compared to commercial libraries and RGBD vision systems.

Finally, we would like to thank all researchers having submitted papers to this special issue and we hope to see many of these contributions published in future issues. 